Research Paper

\title{
Tie2 Regulates Tumor Metastasis of Oral Squamous Cell Carcinomas
}

\author{
Daisuke Kitajima $^{1}$, Atsushi Kasamatsu ${ }^{2}$, Dai Nakashima ${ }^{1}$, Isao Miyamoto ${ }^{1}$, Yasushi Kimura ${ }^{1}$, Tomoaki \\ Saito', Takane Suzuki³, Yosuke Endo-Sakamoto², Masashi Shiiba4, Hideki Tanzawa, ${ }^{1,2}$, and Katsuhiro \\ Uzawa ${ }^{1,2}$ \\ 1. Department of Oral Science Graduate School of Medicine, Chiba University, 1-8-1 Inohana, Chuo-ku, Chiba 260-8670, Japan; \\ 2. Department of Dentistry and Oral-Maxillofacial Surgery, Chiba University Hospital, 1-8-1 Inohana, Chuo-ku, Chiba 260-8670, Japan; \\ 3. Department of Environmental Health Science and \\ 4. Department of Clinical Oncology, Graduate School of Medicine, Chiba University, 1-8-1 Inohana, Chuo-ku, Chiba 260-8670, Japan. \\ $\bowtie$ Corresponding authors: E-mail: kasamatsua@faculty.chiba-u.jp (AK) and uzawak@faculty.chiba-u.jp (KU).
}

(C) Ivyspring International Publisher. Reproduction is permitted for personal, noncommercial use, provided that the article is in whole, unmodified, and properly cited. See http://ivyspring.com/terms for terms and conditions.

Received: 2015.09.11; Accepted: 2016.01.22; Published: 2016.03.18

\begin{abstract}
The endothelial-specific receptor, tyrosine kinase with immunoglobulin-like loops and epidermal growth factor homology domains-2 (Tie2) is a member of the tyrosine kinase family and is ubiquitous in normal tissues; however, little is known about the mechanisms and roles of Tie2 in oral squamous cell carcinomas (OSCCs). In the current study, we investigated the expression status of Tie2 in OSCCs by quantitative reverse transcriptase-polymerase chain reaction, immunoblotting, and immunohistochemistry and the functional mechanisms of Tie2 using its overexpressed OSCC (oeTie2) cells and Tie2 blocking by its antibody. We found that Tie2 expression was down-regulated significantly $(p<0.05)$ in OSCCs compared with normal counterparts in vitro and in vivo. Interestingly, oeTie2 cells showed higher cellular adhesion $(p<0.05)$ and lower cellular invasion $(p<0.05)$ compared with control cells; whereas there was similar cellular proliferation in both transfectants. Furthermore, cellular adhesion was inhibited and invasion was activated by Tie 2 function-blocking antibody $(p<0.05)$, indicating that Tie2 directly regulates cellular adhesion and invasion. As expected, among the clinical variables analyzed, Tie2-positivity in patients with OSCC was correlated closely with negative lymph node metastasis. These results suggested for the first time that Tie2 plays an important role in tumor metastasis and may be a potential biomarker for OSCC metastasis.
\end{abstract}

Key words: Tie2, Oral Cancer

\section{Introduction}

Tyrosine kinase with immunoglobulin-like loops and epidermal growth factor homology (Tie) domains, i.e., Tie1 and Tie2, is a member of the endothelial-specific receptor tyrosine kinase (RTK) [1, 2], which is essential for formation of embryonic vasculature [3]. Tie2 maintains the vascular integrity of mature vessels by enhancing endothelial barrier function [4-8]. Gene-targeting analyses of Tie2 have identified the necessity for these vascular endothelial RTKs for neovascular formation in ischemic diseases and tumors [9]. However, Tie1 is expressed exclusively in endothelial cells, but its role is incompletely defined.

Tie2 is tyrosine phosphorylated in normal adult endothelium tissues and expressed in the endothelium of neovessels of regenerating organs and several types of tumor, including leukemia, breast, gastric, and thyroid cancers [10-15]. There is, however, little information about Tie2 function in oral squamous cell carcinomas (OSCCs).

The current study found for the first time that Tie2 expression is correlated closely with regional lymph node metastasis in OSCCs. Processes involved in tumor metastasis include loss of cell-cell and/or 
cell-extracellular matrix (ECM) adhesion, which allows these cells to migrate from their site of origin, degrade the ECM, acquire motility, and invade phenotypes leading to metastases [16-18]. We found that Tie2 expression was significantly lower in OSCCs than in normal counterparts in vitro and in vivo and that a model of Tie2 overexpression showed novel Tie2 functions in OSCCs. Our results indicated that Tie2 may be a potential therapeutic target for patients with OSCC.

\section{Materials and Methods}

\section{Ethics statement}

The Ethics Committee of the Graduate School of Medicine, Chiba University approved the study protocol (approval number, 236), which was performed according to the tenets of the Declaration of Helsinki. All patients provided written informed consent.

\section{OSCC-derived cell lines and tissue specimens}

OSCC-derived cell lines (HSC-2, HSC-3, HSC-4, KOSC-2, Sa3, Ca9-22, SAS, HO-1-N-1, and HO-1-u-1) were obtained from the Human Science Research Resources Bank (Osaka, Japan) or the RIKEN BioResource Center (Tsukuba, Japan) through the National Bio-Resource Project of the Ministry of Education, Culture, Sports, Science and Technology. Short tandem repeat profiles confirmed cellular identity. As described in detail previously [19-25], primary cultured human normal oral keratinocytes (HNOKs) were obtained from healthy oral mucosa epithelium specimens collected from young patients at Chiba University Hospital. Three independent HNOKs were primary cultured and maintained in oral keratinocyte medium (ScienCell Research Laboratories, Carlsbad, CA, USA) comprised of $5 \mathrm{ml}$ of oral keratinocyte growth supplement (ScienCell Research Laboratories) and $5 \mathrm{ml}$ of penicillin/streptomycin solution (ScienCell Research Laboratories) [19-25]. OSCC cells were grown in Dulbecco's modified Eagle medium (DMEM) (Sigma-Aldrich, St. Louis, MO, USA) supplemented with 10\% FBS (Sigma-Aldrich) and 50 units $/ \mathrm{ml}$ of penicillin and streptomycin (Sigma-Aldrich). Seventy pairs of primary OSCCs and patient-matched normal oral epithelia were obtained during surgical resections performed at Chiba University Hospital after the patients provided informed consent. The resected tissues were divided into two parts, one of which was frozen immediately and stored at $-80^{\circ} \mathrm{C}$ until RNA isolation, and the second of which was fixed in $20 \%$ buffered formaldehyde solution for pathologic diagnosis and immunohistochemistry (IHC). Histopathologic diagnosis of each tissue was performed according to the World Health Organization criteria at the Department of Pathology of
Chiba University Hospital. Clinicopathological staging was determined based on the tumor-node-metastasis classification of the International Union against Cancer. All patients had histologically confirmed OSCC, and the tumor samples were evaluated to ensure that tumoral tissue was present in more than $80 \%$ of the specimen.

\section{mRNA expression analysis}

Total RNA was isolated using Trizol Reagent (Invitrogen, Carlsbad, CA, USA), according to the manufacturer's instructions. cDNA was generated from $5 \mu \mathrm{g}$ of total RNA using Ready-To-Go You-Prime First-Strand Beads (GE Healthcare, Buckinghamshire, UK) and oligo (dT) primer (Hokkaido System Science, Sapporo, Japan) according to the manufacturers' instructions. As described in detail previously [22], real-time quantitative reverse transcriptase-polymerase chain reaction (qRT-PCR) was performed using the LightCycler 480 apparatus (Roche Diagnostics, Mannheim, Germany). Primers were designed using the Universal ProbeLibrary Assay Design Center (http://lifescience.roche.com/), which specifies the most suitable set. The primer sequences used for qRT-PCR were: Tie2, forward, 5'-CCC CTA TGG GTG TTC CTG T-3'; reverse, 5'-GCT TAC AAT CTG GCC CGT AA-3'; and glyceraldehyde-3-phosphate dehydrogenase (GAPDH), forward, 5'-AAC ATC ATC CCT GCC TCT ACT GG-3'; reverse, 5'-TTG AAG TCA GAG GAG ACC ACT G-3'. The transcript amount was estimated from the respective standard curves and normalized to the GAPDH transcript amount determined in corresponding samples. All samples were analyzed in triplicate, and three independent preparations of RNA were analyzed from each cell line.

\section{Immunoblot analysis}

The cells were washed twice with cold phosphate-buffered saline (PBS) and centrifuged briefly. The cellular pellets were incubated at $4^{\circ} \mathrm{C}$ for 30 minutes in a lysis buffer (7 M urea, $2 \mathrm{M}$ thiourea, $4 \%$ $(\mathrm{w} / \mathrm{v})$ CHAPS, and $10 \mathrm{mM}$ Tris) with a proteinase inhibitor cocktail (Roche Diagnostics). The protein concentration was measured using a commercial Bradford reagent (Bio-Rad, Richmond, CA, USA). Immunoblot analysis was performed described previously [22-25]. Briefly, protein extracts $(20 \mu \mathrm{g})$ were electrophoresed on 4-12\% Bis-Tris gel (Invitrogen), transferred to polyvinylidene fluoride membranes (Invitrogen), and blocked for 1 hour at room temperature in Blocking One (Nacalai Tesque, Kyoto, Japan). The membranes were washed three times with $0.1 \%$ Tween-20 in Tris-buffered saline (TBS-T) and incubated with affinity-purified rabbit anti-Tie2 polyclo- 
nal antibody (Santa Cruz Biotechnology, Santa Cruz, CA, USA) or mouse anti-GAPDH monoclonal antibody (Santa Cruz Biotechnology) overnight at $4^{\circ} \mathrm{C}$. The membranes were washed with TBS-T and incubated with horseradish peroxidase-conjugated anti-rabbit or anti-mouse IgG as a secondary antibody (Promega, Madison, WI, USA) for 1 hour at room temperature. Finally, the membranes were detected using Super-Signal West Pico Chemiluminescent substrate (Thermo Fisher Scientific, Rockford, IL, USA), and immunoblot analyses were visualized by exposing the membranes to BioRad ChemiDoc XRS system (BioRad).The signal intensities were quantitated using Image Lab software (BioRad). Densitometric Tie2 protein data were normalized to GAPDH protein levels.

\section{IHC}

IHC was performed on 4- $\mu$ m sections of paraffin-embedded specimens using rabbit anti-Tie2 polyclonal antibody (Santa Cruz Biotechnology). Briefly, after deparaffinization and hydration, the endogenous peroxidase activity was quenched by a 3-minute incubation in a mixture of $0.3 \%$ hydrogen peroxide solution in $100 \%$ methanol. The sections were blocked for 2 hours at room temperature with 1.5\% blocking serum (Santa Cruz Biotechnology) in PBS before reacting with the anti-Tie2 antibody at $4^{\circ} \mathrm{C}$ in a moist chamber overnight. Upon incubation with the primary antibody, the specimens were washed three times in PBS and treated with Envision reagent (DAKO, Carpinteria, CA, USA) followed by color development in 3,3'-diaminobenzidine tetrahydrochloride (DAKO). The slides then were lightly counterstained with hematoxylin, dehydrated with ethanol, cleaned with xylene, and mounted. Nonspecific binding of the antibodies to other proteins sometimes occurred. As a negative control, triplicate sections were immunostained without exposure to primary antibodies, which confirmed the staining specificity. To quantify the status of Tie2 protein expression in those components, we used the IHC scoring systems described previously [22, 26-29]. The mean percentages of positively stained cells were determined in at least three random fields at $400 \times$ magnification in each section. The intensity of the Tie 2 immunoreaction was scored as follows: $0+$, none; $1+$, weak; $2+$, moderate; and $3+$, intense. The cellular numbers and the staining intensities were multiplied to produce Tie2 IHC scores. To determine the cutoff points of Tie2 IHC scores, we analyzed the IHC scores of 70 patients using the receiver operating characteristic curve (ROC) and Youden index. Cases with a score over 72.5 (ROC curve and Youden index for normal tissue) were considered Tie2-negative. Two inde- pendent pathologists from Chiba University Hospital, neither of whom had knowledge of the patients' clinical status, made these judgments.

\section{Transfection of Tie2 overexpression vector}

OSCC-derived cells (SAS and Sa3) were transfected with Tie2 vector designed to cause overexpression of Tie 2 cDNA (oeTie2 cells) (OriGene Technologies, Rockville, MD, USA) and non-targeting vector (Mock cells) (OriGene Technologies) using Lipofectamine 3000 (Invitrogen). After transfection, the cells that stably overexpressed Tie2 were isolated by the selection marker, G-418 Solution (Roche Diagnostics). Two to three weeks after incubation with G-418 Solution, individual clones were isolated and used for further experiments.

\section{Proliferation assay}

To investigate the effect of Tie2 overexpression on cellular proliferation, oeTie 2 and Mock cells were seeded in six-well plates at a density of $1 \times 10^{4}$ viable cells/well. At the indicated time points, the cells were trypsinized and counted in triplicate using a hemocytometer[22-25].

\section{Adhesion assay}

Collagen I-coated 96-well plates were incubated for 1 hour at $37^{\circ} \mathrm{C}$. Adhesion assay was preformed described previously [23]. Briefly, the cells were seeded at a density of $2 \times 10^{4}$ cells/well and incubated for 1 hour in DMEM, washed once with PBS, fixed in methanol, stained with crystal violet, and photographed. The numbers of stained cells were measured using a microplate spectrophotometer (absorbance at $540 \mathrm{~nm}$ and at $405 \mathrm{~nm}$ to subtract the background).

\section{Invasion assay}

Invasion assay was preformed described previously [23]. Briefly, a total of $2.5 \times 10^{5}$ cells resuspended in serum-free medium were seeded on a polyethylene terephthalate membrane insert with a pore size of 8 $\mu \mathrm{m}$ in a trans-well apparatus (Becton Dickinson, Franklin Lakes, NJ, USA). Two milliliters of DMEM with $10 \%$ FBS was added to the lower chamber. After the cells were incubated for 72 hours at $37^{\circ} \mathrm{C}$, the insert was washed with PBS, and cells on the top surface of the insert were removed with a cotton swab. Cells adhering to the lower surface of the membrane were stained with crystal violet/methanol, and the number of cells entering the pores in five random fields was counted using a light microscope at $100 \times$ magnification.

\section{Inhibition of cellular adhesion and invasion by blocking Tie2}

The Tie 2 overexpressed cells and control cells 
were resuspended in serum-free medium. The cellular suspension was incubated with the indicated concentrations of Tie2 polyclonal antibody (Santa Cruz Biotechnology) for 30 minutes at room temperature. The cells were seeded and cellular adhesion and invasion assays were performed.

\section{Statistical analysis}

To compare Tie2 expression levels, statistical significance was evaluated using the Mann-Whitney U-test. The relationships between the Tie2 IHC scores and clinicopathological profiles were evaluated using the $\chi^{2}$ test, Fisher's exact test, and Mann-Whitney U-test. $P<0.05$ was considered significant. The data are expressed as the mean \pm standard error of the mean. Survival curves were obtained by the Kaplan-Meier method, and differences in survival rates between Tie2-positive and Tie2-negative cases were compared by log-rank test with $95 \%$ significance.

\section{Results}

\section{Down-regulation of Tie2 in OSCC-derived cell lines}

To investigate the expression status of Tie2, identified as a cancer-related gene by our previous microarray data [30], we performed qRT-PCR and immunoblot analyses using nine OSCC-derived cell lines (HSC-2, HSC-3, HSC-4, KOSC-2, Sa3, Ca9-22, SAS, HO-1-N-1, and HO-1-u-1) and HNOKs. Tie2 mRNA was down-regulated significantly $(p<0.05)$ in all OSCC-derived cell lines compared with the HNOKs (Fig. 1A). Fig. 1B shows representative results of immunoblot analysis. The Tie2 protein expression decreased significantly $(p<0.05)$ in all OSCC-derived cell lines compared with the HNOKs.

\section{Evaluation of Tie2 expression in primary OSCCs}

We analyzed the Tie2 protein expression in primary OSCCs using the IHC scoring system [19, 22, 26-29, 31, 32]. The Tie2 IHC scores in normal oral tissues and OSCCs ranged from 65.0 to 125.0 (median, 95.0 ) and 20.0 to 90.0 (median, 58.75), respectively. The IHC scores in primary OSCCs were significantly $(p<0.05)$ lower than in normal oral tissues (Fig. 2A). Representative IHC results for Tie2 protein in normal oral tissue and primary OSCC are shown in Fig. 2B and $\mathrm{C}$, respectively. Strong Tie2 immunoreactivity was detected in normal tissue, whereas the OSCC tissue showed almost negative immunostaining (Fig. 2C). To determine an optimal cutoff point of the identified IHC scores, we used the ROC curve analysis and Youden index. ROC curve analysis (area under the curve, 0.95132; 95\% confidence interval,
$0.9219-0.9807 ; p<0.05)$ and Youden index (sensitivity, $78.6 \%$; specificity, $95.7 \%, p<0.05$ ) showed that the cutoff value was 72.5. (Fig. 2D, E).

\section{Establishment of Tie2 overexpressed cells}

Since frequent down-regulation of Tie 2 was observed in OSCC in vitro and in vivo (Figs. 1 and 2), the OSCC-derived cells (SAS and Sa3) were transfected with oeTie2 and non-targeting vector (Mock) to investigate the Tie2 function in OSCC cells. To assess the efficiency of the transfection, we performed qRT-PCR and immunoblot analyses. The Tie2 mRNA expression in the oeTie 2 cells was significantly $(p<$ 0.05) higher than in Mock cells (Fig. 3A). The Tie2 protein level in the oeTie2 cells also increased compared with Mock cells (Fig. 3B).
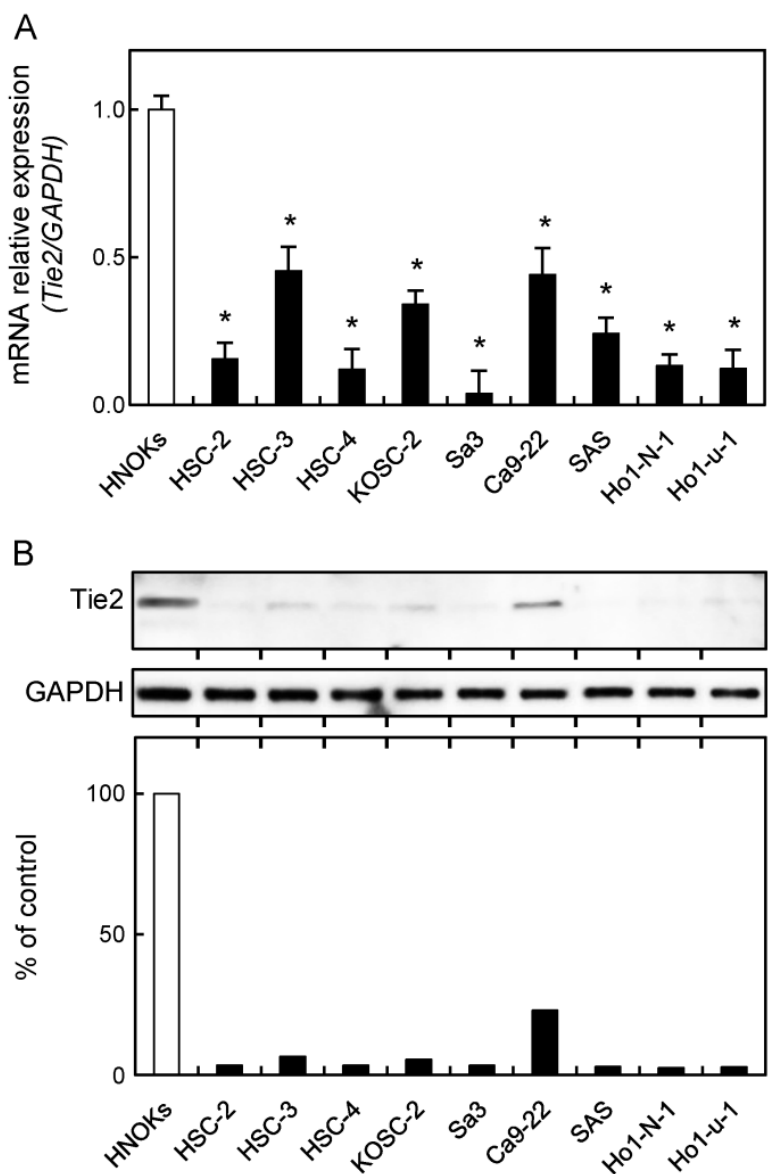

Figure 1. Down-regulation of Tie2 expression in OSCC-derived cell lines. (A) Quantification of Tie2 mRNA expression in OSCC-derived cell lines by qRT-PCR analysis. Significant down-regulation of Tie 2 mRNA is seen in nine OSCC-derived cell lines compared with HNOKs $\left(*_{p}<0.05\right.$, Mann-Whitney $U$-test). Data are expressed as the means \pm standard errors of the mean of triplicate results. (B) Immunoblot analysis of Tie2 protein in OSCC-derived cell lines and HNOKs. Tie2 protein expression is down-regulated in OSCC-derived cell lines compared with HNOKs. Densitometric Tie2 protein data are normalized to GAPDH protein levels. The values are expressed as a percentage of HNOKs. 

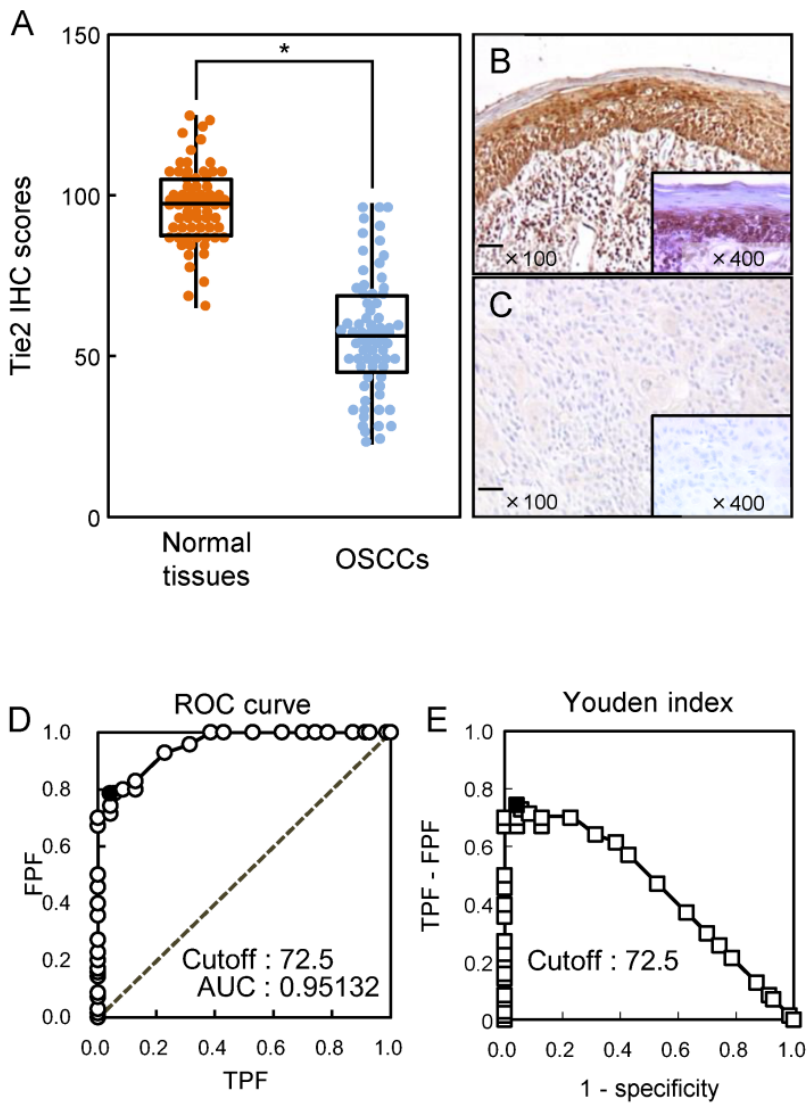

Figure 2. Evaluation of Tie2 expression in primary OSCCs. (A) The Tie2 IHC scores of normal oral tissues and OSCCs range from 65.0 to 125.0 (median, 95.0) and 20.0 to 90.0 (median, 58.75), respectively. The IHC scores in primary OSCCs are significantly $\left({ }^{*} p<0.05\right.$, Mann-Whitney U-test) lower than in normal oral tissues. Representative IHC results for Tie2 protein in normal oral tissue (B) and primary OSCC (C). Strong Tie2 immunoreactivity is seen in normal oral tissues, whereas the OSCCs show almost negative immunostaining. Original magnification, $X 100$. Scale bars, $100 \mu \mathrm{m}$. (D) The ROC curve analysis shows that the optimal cutoff point is 72.5 (area under the curve, $0.95132 ; 95 \%$ confidence interval, 0.9219-0.9807; $p<0.05$ ). (E) Youden Index analysis shows that the optimal cutoff point is 72.5 (sensitivity, $78.6 \%$; specificity, 95.7\%, $p<0.05$ ).
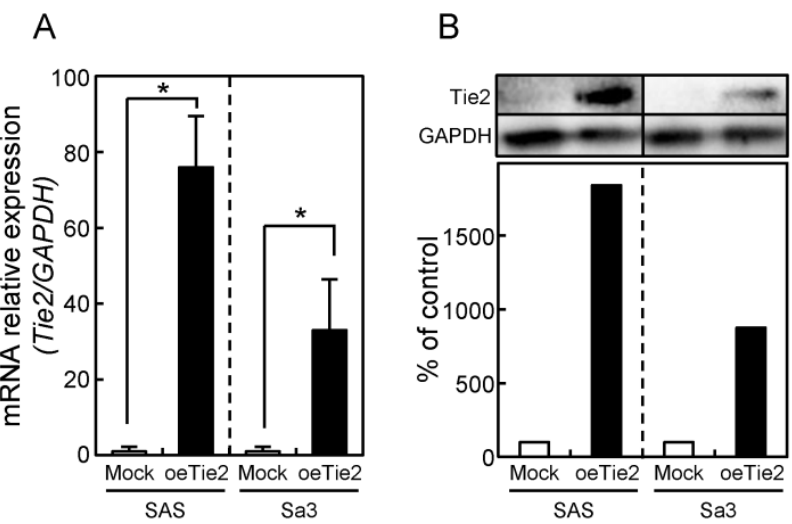

Figure 3. Establishment of Tie2 overexpressed cells. Significant $\left({ }^{*} p<\right.$ 0.05, Mann-Whitney U-test) up-regulation of Tie2 mRNA (A) and protein (B) are observed in oeTie2 cells compared with Mock cells using QRT-PCR and immunoblot analyses. Densitometric Tie2 protein data are normalized to GAPDH protein levels. The values are expressed as a percentage of the Mock cells.
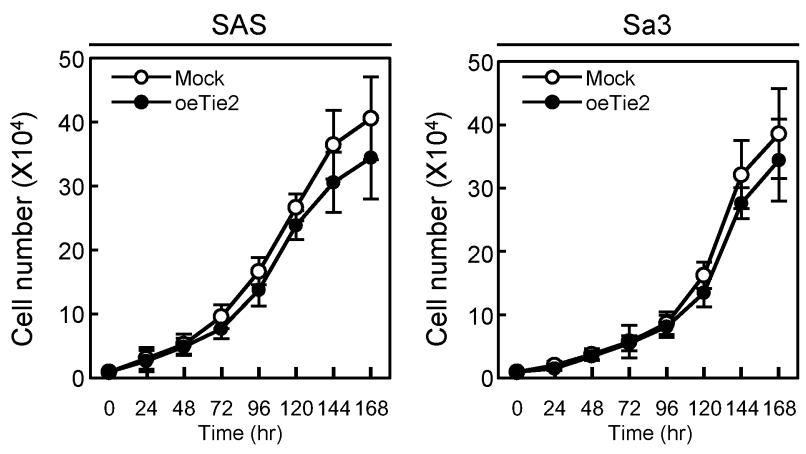

Figure 4. Cellular proliferation in Tie2 overexpressed cells. To determine the effect of Tie2 overexpression on cellular proliferation, oeTie2 and Mock cells are seeded in six-well plates at a density of $1 \times 10^{4}$ viable cells/well. Both transfectants are counted on seven consecutive days. oeTie2 cells (SASand Sa3-derived transfectants; 2 clones each) do not affect cellular growth compared with Mock cells after 7 days (168 hours [hr]). The results are expressed as the means \pm standard errors of the mean of values from three assays.

\section{Functional analyses of Tie2 overexpressed cells}

We performed a cellular proliferation assay to evaluate the effect of Tie2 overexpression on cellular growth. The Mock and oeTie2 cells had similar growth curves, indicating that overexpression of Tie2 did not affect cellular growth (Fig. 4). We then carried out cellular adhesion and invasion assays to determine the biologic effects of Tie2 in SAS and Sa3 cells (Figs. 5, 6, respectively).

Cellular adhesion in the oeTie2 cells significantly increased $(p<0.05)$ compared with the Mock cells (Fig. 5A). We further examined whether the adhesion activity of Tie2 overexpressed cells varied when Tie2 was blocked. The adhesion activity of oeTie 2 cells treated with Tie2 polyclonal antibody was inhibited dramatically in a dose-dependent manner (Fig. 5B, C). In addition. oeTie2 cells showed decreased cellular invasion compared with Mock cells (Fig. 6A; $p<0.05$ ). After treatment with Tie2 polyclonal antibody, invasion of oeTie 2 cells was activated significantly compared with the untreated cells (Fig. 6B), suggesting that Tie2 may directly regulate cellular adhesion and invasion.

\section{Correlation between Tie2 expression and clinical classifications of OSCCs}

The correlations between the clinicopathologic characteristics of patients with OSCC and their Tie2 protein levels using the IHC scoring system are shown in Table 1 . Among the clinical classifications, Tie2-negative patients exhibited significantly $(p<$ 0.01 ) more regional lymph node metastasis compared with Tie2-positive patients. Survival analysis using the Kaplan-Meier method showed that Tie2 expression was not significantly related to overall survival $(p$ $=0.178$; Fig. S1). 
Table 1. Correlation between Tie2 expression and clinical classification in OSCCs.

\begin{tabular}{|c|c|c|c|c|}
\hline \multirow[t]{2}{*}{ Clinical classification } & \multirow[t]{2}{*}{ Total } & \multicolumn{2}{|c|}{$\begin{array}{l}\text { Immunostaining Results } \\
\text { No. patients }\end{array}$} & \multirow[t]{2}{*}{$p$ value } \\
\hline & & Tie & Tie2-positive & \\
\hline \multicolumn{5}{|l|}{ Age at surgery (years) } \\
\hline$<60$ & 18 & 14 & 4 & \\
\hline$\geqq 60,<70$ & 20 & 13 & 7 & $0.788^{a}$ \\
\hline$\geqq 70$ & 32 & 25 & 7 & \\
\hline \multicolumn{5}{|l|}{ Gender } \\
\hline Male & 47 & 34 & 13 & $0.773^{b}$ \\
\hline Female & 23 & 18 & 5 & \\
\hline \multicolumn{5}{|l|}{ T-primary tumor } \\
\hline $\mathrm{T} 1$ & 5 & 3 & 2 & $0.051^{\mathrm{c}}$ \\
\hline $\mathrm{T} 2$ & 48 & 33 & 15 & \\
\hline T3 & 9 & 8 & 1 & \\
\hline $\mathrm{T} 4$ & 8 & 8 & 0 & \\
\hline \multicolumn{5}{|l|}{ N-regional lymph node } \\
\hline Negative & 53 & 35 & 18 & $0.008 \mathrm{~b}^{* *}$ \\
\hline Positive & 17 & 17 & 0 & \\
\hline \multicolumn{5}{|l|}{ Vascular invasion } \\
\hline Negative & 53 & 37 & 16 & $0.203^{c}$ \\
\hline Positive & 17 & 15 & 2 & \\
\hline \multicolumn{5}{|l|}{ Stage } \\
\hline I & 3 & 1 & 2 & $0.006^{* *_{c}}$ \\
\hline II & 49 & 28 & 21 & \\
\hline III & 19 & 15 & 4 & \\
\hline IV & 44 & 24 & 20 & \\
\hline \multicolumn{5}{|l|}{ Histopathologic type } \\
\hline Well & 54 & 38 & 16 & $0.175^{c}$ \\
\hline Moderately & 13 & 11 & 2 & \\
\hline Poorly & 3 & 3 & 0 & \\
\hline \multicolumn{5}{|l|}{ Tumoral site } \\
\hline Tongue & 47 & 35 & 12 & $0.846^{c}$ \\
\hline Gingiva & 14 & 9 & 5 & \\
\hline Buccal mucosa & 3 & 3 & 0 & \\
\hline Soft palate & 3 & 2 & 1 & \\
\hline Oral floor & 3 & 3 & 0 & \\
\hline
\end{tabular}

\section{Discussion}

Tie2 was down-regulated in OSCC in vitro and in vivo and that Tie2 controls cellular adhesion and invasion activities. As expected, no metastases to regional lymph nodes occurred in patients with OSCC who were Tie2-positive (Table $1, p<0.05$ ).

Although Tie2 first was identified as an endothelial cell-specific receptor for vascular remodeling and stabilization signals in angiogenesis [3, 16, 33-38], research has shown the presence of RTK in the non-stromal cell population of cancers $[17,18,38]$ such as leukemia and gastric, thyroid, and breast cancers [15, 39-41]. Tie2 was overexpressed in gastric, breast, and hepatocellular cancers and related to tumor angiogenesis $[42,43]$. In contrast, the current results showed that Tie2 was down-regulated in OSCCs; therefore, Tie2 plays pivotal roles in tumor metastasis of several types of cancers.
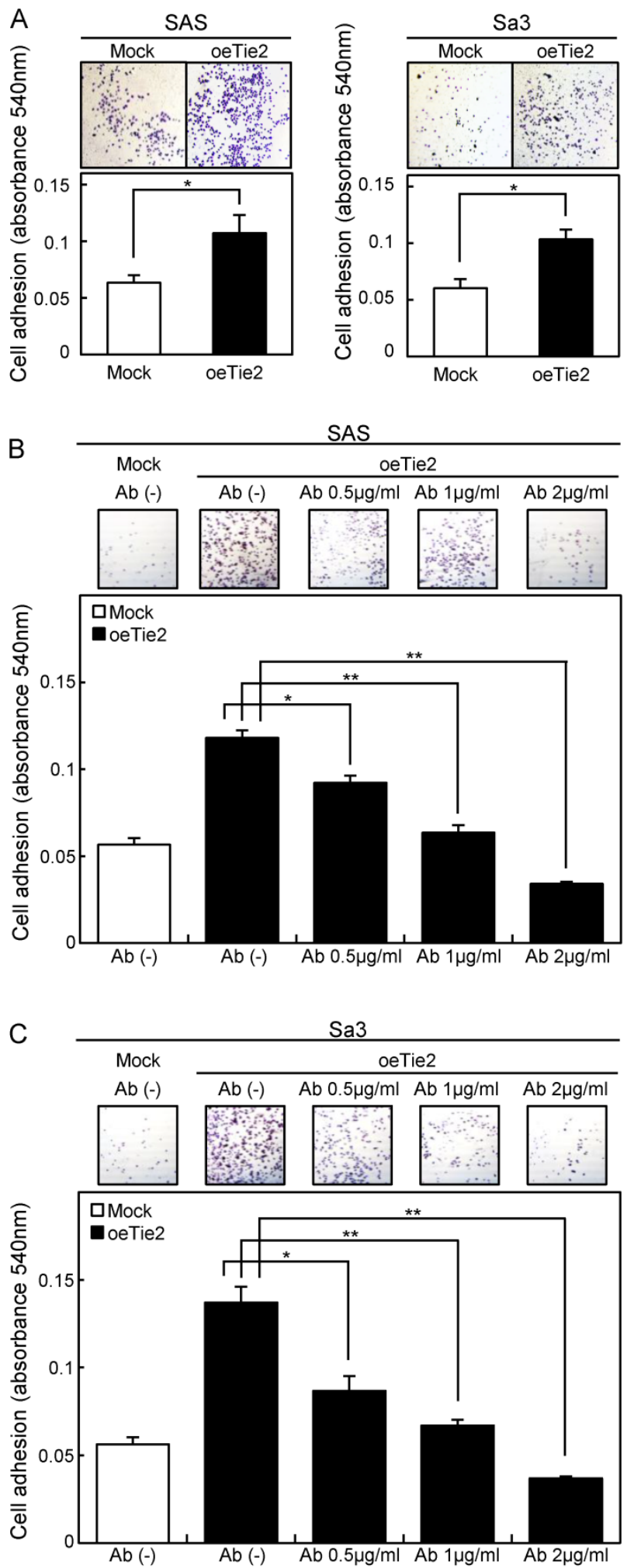

Figure 5. Adhesion assay of Tie2 overexpressed cells. (A) Adhesion assay of Tie2 overexpressed cells. To evaluate the adhesion ability, oeTie2 and Mock cells are seeded on collagen I-coated 96-well plates at a density of $2 \times 10^{4}$ cells/well and allowed to adhere for 1 hour. After crystal violet staining, the numbers of stained cells are measured using a microplate spectrophotometer (absorbance at $540 \mathrm{~nm}$ and at $405 \mathrm{~nm}$ to subtract background). The cellular adhesion of the oeTie2 cells increased significantly $(p<0.05)$ compared with the Mock cells. (B, C) Inhibition of cellular adhesion by Tie2 antibody, oeTie2 cells are treated with Tie2 antibody before the adhesion assay. The numbers of oeTie 2 cells attached to the dishes are decreased in a dose-dependent manner of the Tie2 antibody (B, SAS cells; C, Sa3 cells). 
A
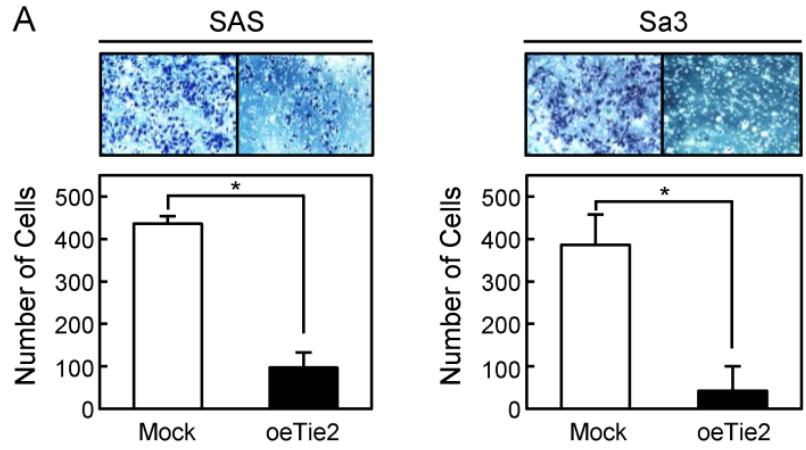

B
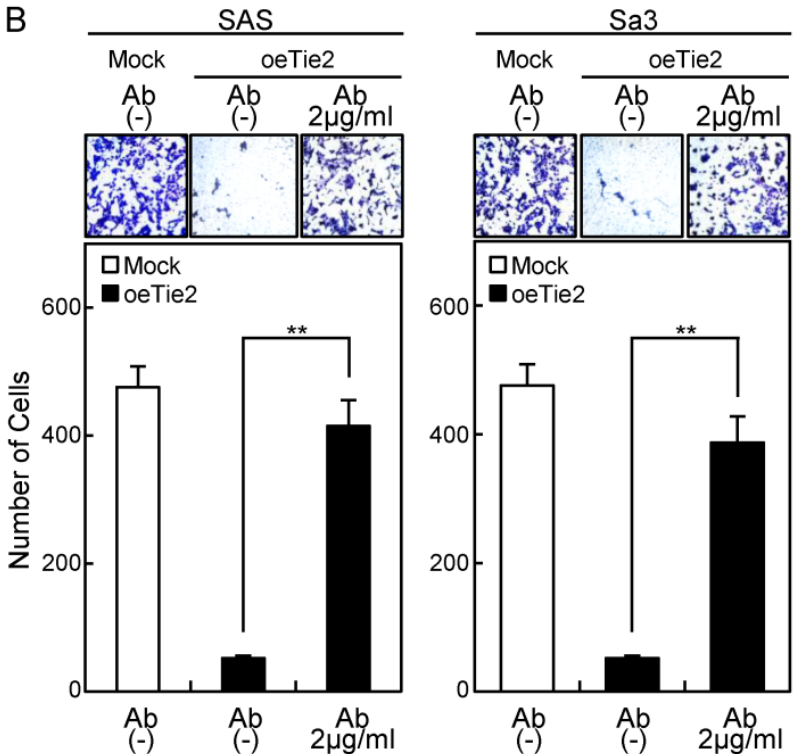

Figure 6. Invasion assay of Tie2 overexpressed cells. (A) Invasion assay of Tie2 overexpressed cells. After crystal violet staining, the numbers of cells invading the pores are counted ( $\times 100$ magnification). The numbers of penetrating oeTie2 cells are significantly lower than with Mock cells $(* p<0.05$, Mann-Whitney $U$ test). (B) Activation of cellular invasion by Tie2 antibody. The oeTie2 cells are treated with Tie2 antibody before the invasion assay. The numbers of oeTie2 cells invading the pores increase after treatment with Tie2 antibody $(2 \mu \mathrm{g} / \mathrm{ml})(B$, SAS cells; C, Sa3 cells, $* * p<0.01)$.

In the current study, we explored the anti-metastasis activities of Tie2 in OSCC, because Tie2 expression in OSCC was correlated significantly with lymph node metastasis using our IHC scoring system (Table 1). Cancer metastasis requires loss of cell-cell and cell-ECM adhesions and invasion through the ECM. Cellular adhesion is maintained in epithelial tissues by the adherens junction, which is down-regulated in many cancers to promote transformation and may profoundly affect cellular motility and differentiation [44-48]. ECM degradation decreases cellular adhesion and increases cellular invasion [49], indicating that the ECM functions are not limited to a structural role but may affect multiple and diverse functions of cancer cells.
The cellular adhesion activity of Tie2 had been reported in human umbilical vein endothelial cells [50]. Consistent with that study, our functional analyses indicated that Tie2-overexpressed cells acquired self-sustained cellular adhesion and decreased invasion phenotypes, suggesting that Tie2 would be, in part, a key modulator of OSCC tumor adhesion and invasion. Although the biologic meaning of these molecular interactions remains to be determined, Tie2 seems to have complex regulatory mechanisms, especially in oral cancer metastasis. In addition to a previous study in which patients with angiosarcoma with high expression of Tie2 had a good prognosis [51], no metastases occurred in patients with Tie2-positive OSCC.

We speculated that Tie2 expression levels are correlated closely with vascular invasion, because our data indicated that Tie 2 controls cellular adhesion and invasion. Consistent with that hypothesis, patients with OSCC who were Tie2-positive tended to have high vascular invasion. However, there was no significant difference between Tie2-positivity and Tie2-negativity (Table $1, p<0.203$ ).

In conclusion, using several approaches, we showed that Tie2 is expressed not only in the human vascular system but also in human normal oral tissue and OSCC. These results, together with the fact that OSCCs express low levels of Tie2, suggested that a previously unknown Tie2 function in OSCC cells controls critical aspects of the metastatic behavior of OSCCs. This provides new insight into the highly metastatic phenotype of OSCC. While further studies are needed to study Tie2, the current data suggested that Tie2 plays an important role in cellular adhesion and invasion and may be a potential biomarker for OSCCs.

\section{Supplementary materials}

Figure S1. http:/ / www.jcancer.org/v07p0600s1.pdf

\section{Abbreviations}

Tie2: The endothelial-specific receptor, tyrosine kinase with immunoglobulin-like loops and epidermal growth factor homology domains-2; OSCCs: oral squamous cell carcinomas; RTK: receptor tyrosine kinase; ECM: extracellular matrix; HNOKs: human normal oral keratinocytes; oeTie2: overexpression of Tie2.

\section{Acknowledgments}

We thank Ms. Lynda C. Charters for editing this manuscript. The authors received no financial support. 


\section{Competing Interests}

The authors have no conflicts of interest to disclose.

\section{References}

1. Schnurch H, Risau W. Expression of tie-2, a member of a novel family of receptor tyrosine kinases, in the endothelial cell lineage. Development. 1993; 119: 957-68.

2. Maisonpierre PC, Goldfarb M, Yancopoulos GD, Gao G. Distinct rat genes with related profiles of expression define a TIE receptor tyrosine kinase family. Oncogene. 1993; 8: 1631-7.

3. Sato TN, Tozawa Y, Deutsch U, Wolburg-Buchholz K, Fujiwara Y, Gendron-Maguire $\mathrm{M}$, et al. Distinct roles of the receptor tyrosine kinases Tie-1 and Tie-2 in blood vessel formation. Nature. 1995; 376: 70-4.

4. Mammoto T, Parikh SM, Mammoto A, Gallagher D, Chan B, Mostoslavsky G, et al. Angiopoietin-1 requires p190 RhoGAP to protect against vascular leakage in vivo. J Biol Chem. 2007; 282: 23910-8.

5. Jho D, Mehta D, Ahmmed G, Gao XP, Tiruppathi C, Broman M, et al. Angiopoietin-1 opposes VEGF-induced increase in endothelial permeability by inhibiting TRPC1-dependent Ca2 influx. Circ Res. 2005; 96: 1282-90.

6. Brindle NP, Saharinen P, Alitalo K. Signaling and functions of angiopoietin-1 in vascular protection. Circ Res. 2006; 98: 1014-23.

7. Baffert F, Le T, Thurston G, McDonald DM. Angiopoietin-1 decreases plasma leakage by reducing number and size of endothelial gaps in venules. Am J Physiol Heart Circ Physiol. 2006; 290: H107-18.

8. Gamble JR, Drew J, Trezise L, Underwood A, Parsons M, Kasminkas L, et al. Angiopoietin-1 is an antipermeability and anti-inflammatory agent in vitro and targets cell junctions. Circ Res. 2000; 87: 603-7.

9. Yancopoulos GD, Davis S, Gale NW, Rudge JS, Wiegand SJ, Holash J. Vascular-specific growth factors and blood vessel formation. Nature. 2000; 407: 242-8.

10. Peters KG, Kontos CD, Lin PC, Wong AL, Rao P, Huang L, et al. Functional significance of Tie 2 signaling in the adult vasculature. Recent Prog Horm Res. 2004; 59: 51-71.

11. Wong AL, Haroon ZA, Werner S, Dewhirst MW, Greenberg CS, Peters KG. Tie2 expression and phosphorylation in angiogenic and quiescent adult tissues. Circ Res. 1997; 81: 567-74

12. Kukk E, Wartiovaara U, Gunji Y, Kaukonen J, Buhring HJ, Rappold I, et al. Analysis of Tie receptor tyrosine kinase in haemopoietic progenitor and leukaemia cells. Br J Haematol. 1997; 98: 195-203.

13. Wang J, Wu K, Zhang D, Tang H, Xie H, Hong L, et al. Expressions and clinical significances of angiopoietin-1, -2 and Tie2 in human gastric cancer. Biochem Biophys Res Commun. 2005; 337: 386-93.

14. Shirakawa K, Tsuda H, Heike $Y$, Kato K, Asada R, Inomata M, et al. Absence of endothelial cells, central necrosis, and fibrosis are associated with aggressive inflammatory breast cancer. Cancer Res. 2001; 61: 445-51.

15. Mitsutake N, Namba H, Takahara K, Ishigaki K, Ishigaki J, Ayabe H, et al. Tie- 2 and angiopoietin- 1 expression in human thyroid tumors. Thyroid. 2002; 12: $95-9$

16. Kim I, Kim HG, So JN, Kim JH, Kwak HJ, Koh GY. Angiopoietin-1 regulates endothelial cell survival through the phosphatidylinositol 3'-Kinase/Akt signal transduction pathway. Circ Res. 2000; 86: 24-9.

17. Lee $\mathrm{OH}, \mathrm{Xu}$ J, Fueyo J, Fuller GN, Aldape KD, Alonso MM, et al. Expression of the receptor tyrosine kinase Tie2 in neoplastic glial cells is associated with integrin beta1-dependent adhesion to the extracellular matrix. Mol Cancer Res. 2006; 4: 915-26.

18. Iwama A, Hamaguchi I, Hashiyama M, Murayama Y, Yasunaga K, Suda T. Molecular cloning and characterization of mouse TIE and TEK receptor tyrosine kinase genes and their expression in hematopoietic stem cells. Biochem Biophys Res Commun. 1993; 195: 301-9.

19. Sakuma K, Kasamatsu A, Yamatoji M, Yamano Y, Fushimi K, Iyoda M, et al. Expression status of Zic family member 2 as a prognostic marker for oral squamous cell carcinoma. J Cancer Res Clin Oncol. 2010; 136: 553-9.

20. Kasamatsu A, Uzawa K, Nakashima D, Koike H, Shiiba M, Bukawa H, et al. Galectin-9 as a regulator of cellular adhesion in human oral squamous cell carcinoma cell lines. Int J Mol Med. 2005; 16: 269-73.

21. Endo Y, Uzawa K, Mochida Y, Shiiba M, Bukawa H, Yokoe H, et al. Sarcoendoplasmic reticulum $\mathrm{Ca}(2+)$ ATPase type 2 downregulated in human oral squamous cell carcinoma. Int J Cancer. 2004; 110: 225-31.

22. Minakawa Y, Kasamatsu A, Koike H, Higo M, Nakashima D, Kouzu Y, et al. Kinesin family member 4A: a potential predictor for progression of human oral cancer. PLoS One. 2013; 8: e85951.

23. Yamatoji M, Kasamatsu A, Kouzu Y, Koike H, Sakamoto Y, Ogawara K, et al. Dermatopontin: a potential predictor for metastasis of human oral cancer. Int J Cancer. 2012; 130: 2903-11.

24. Uchida F, Uzawa K, Kasamatsu A, Takatori H, Sakamoto Y, Ogawara K, et al. Overexpression of cell cycle regulator CDCA3 promotes oral cancer progression by enhancing cell proliferation with prevention of G1 phase arrest. BMC Cancer. 2012; 12: 321
25. Unozawa M, Kasamatsu A, Higo M, Fukumoto C, Koyama T, Sakazume T, et al. Cavin-2 in oral cancer: A potential predictor for tumor progression. Mol Carcinog. 2015.

26. Lombardi DP, Geradts J, Foley JF, Chiao C, Lamb PW, Barrett JC. Loss of KAI1 expression in the progression of colorectal cancer. Cancer Res. 1999; 59: 5724-31.

27. Shimada K, Uzawa K, Kato M, Endo Y, Shiiba M, Bukawa H, et al. Aberrant expression of RAB1A in human tongue cancer. Br J Cancer. 2005; 92: 1915-21.

28. Baba T, Sakamoto Y, Kasamatsu A, Minakawa Y, Yokota S, Higo M, et al. Persephin: A potential key component in human oral cancer progression through the RET receptor tyrosine kinase-mitogen-activated protein kinase signaling pathway. Mol Carcinog. 2015; 54: 608-17.

29. Ishige S, Kasamatsu A, Ogoshi K, Saito Y, Usukura K, Yokoe H, et al. Decreased expression of kallikrein-related peptidase 13: possible contribution to metastasis of human oral cancer. Mol Carcinog. 2014; 53: 557-65.

30. Yamano Y, Uzawa K, Shinozuka K, Fushimi K, Ishigami T, Nomura H, et al. Hyaluronan-mediated motility: a target in oral squamous cell carcinoma. Int J Oncol. 2008; 32: 1001-9.

31. Kimura R, Kasamatsu A, Koyama T, Fukumoto C, Kouzu Y, Higo M, et al. Glutamate acid decarboxylase 1 promotes metastasis of human oral cancer by beta-catenin translocation and MMP7 activation. BMC Cancer. 2013; 13: 555.

32. Kouzu Y, Uzawa K, Koike H, Saito K, Nakashima D, Higo M, et al. Overexpression of stathmin in oral squamous-cell carcinoma: correlation with tumour progression and poor prognosis. Br J Cancer. 2006; 94: 717-23.

33. Augustin HG, Koh GY, Thurston G, Alitalo K. Control of vascular morphogenesis and homeostasis through the angiopoietin-Tie system. Nat Rev Mol Cell Biol. 2009; 10: 165-77.

34. Holopainen T, Huang H, Chen C, Kim KE, Zhang L, Zhou F, et al. Angiopoietin-1 overexpression modulates vascular endothelium to facilitate tumor cell dissemination and metastasis establishment. Cancer Res. 2009; 69: 4656-64.

35. Arai F, Hirao A, Ohmura M, Sato H, Matsuoka S, Takubo K, et al. Tie2/angiopoietin-1 signaling regulates hematopoietic stem cell quiescence in the bone marrow niche. Cell. 2004; 118: 149-61.

36. Dumont DJ, Gradwohl G, Fong GH, Puri MC, Gertsenstein M, Auerbach A, et al. Dominant-negative and targeted null mutations in the endothelial receptor tyrosine kinase, tek, reveal a critical role in vasculogenesis of the embryo. Genes Dev. 1994; 8: 1897-909.

37. Suri $C$, Jones PF, Patan $S$, Bartunkova $S$, Maisonpierre PC, Davis $S$, et al. Requisite role of angiopoietin-1, a ligand for the TIE2 receptor, during embryonic angiogenesis. Cell. 1996; 87: 1171-80.

38. Sato TN, Qin Y, Kozak CA, Audus KL. Tie-1 and tie-2 define another class of putative receptor tyrosine kinase genes expressed in early embryonic vascular system. Proc Natl Acad Sci U S A. 1993; 90: 9355-8.

39. Dales JP, Garcia S, Bonnier P, Duffaud F, Meunier-Carpentier S, Andrac-Meyer L, et al. Tie2/Tek expression in breast carcinoma: correlations of immunohistochemical assays and long-term follow-up in a series of 909 patients. Int J Oncol. 2003; 22: 391-7.

40. Liu D, Martin V, Fueyo J, Lee $\mathrm{OH}, \mathrm{Xu}$ J, Cortes-Santiago N, et al. Tie2/TEK modulates the interaction of glioma and brain tumor stem cells with endothelial cells and promotes an invasive phenotype. Oncotarget. 2010; 1: 700-9.

41. Cho $\mathrm{CH}$, Kammerer RA, Lee HJ, Steinmetz MO, Ryu YS, Lee SH, et al. COMP-Ang1: a designed angiopoietin-1 variant with nonleaky angiogenic activity. Proc Natl Acad Sci U S A. 2004; 101: 5547-52.

42. Min Y, Ren X, Vaught DB, Chen J, Donnelly E, Lynch CC, et al. Tie2 signaling regulates osteoclastogenesis and osteolytic bone invasion of breast cancer. Cancer Res. 2010; 70: 2819-28.

43. Tanaka S, Sugimachi K, Yamashita Yi Y, Ohga T, Shirabe K, Shimada M, et al. Tie2 vascular endothelial receptor expression and function in hepatocellular carcinoma. Hepatology. 2002; 35: 861-7.

44. Jamora C, Fuchs E. Intercellular adhesion, signalling and the cytoskeleton. Nat Cell Biol. 2002; 4: E101-8.

45. Green KJ, Simpson CL. Desmosomes: new perspectives on a classic. J Invest Dermatol. 2007; 127: 2499-515.

46. Dusek RL, Attardi LD. Desmosomes: new perpetrators in tumour suppression. Nat Rev Cancer. 2011; 11: 317-23.

47. South AP, Wan H, Stone MG, Dopping-Hepenstal PJ, Purkis PE, Marshall JF, et al. Lack of plakophilin 1 increases keratinocyte migration and reduces desmosome stability. J Cell Sci. 2003; 116: 3303-14.

48. Yin T, Green KJ. Regulation of desmosome assembly and adhesion. Semin Cell Dev Biol. 2004; 15: 665-77.

49. Ding KF, Sun LF, Ge WT, Hu HG, Zhang SZ, Zheng S. Effect of SNC19/ST14 gene overexpression on invasion of colorectal cancer cells. World J Gastroenterol. 2005; 11: 5651-4

50. Saharinen P, Eklund L, Miettinen J, Wirkkala R, Anisimov A, Winderlich M, et al. Angiopoietins assemble distinct Tie2 signalling complexes in endothelial cell-cell and cell-matrix contacts. Nat Cell Biol. 2008; 10: 527-37.

51. Buehler D, Rush P, Hasenstein JR, Rice SR, Hafez GR, Longley BJ, et al. Expression of angiopoietin-TIE system components in angiosarcoma. Mod Pathol. 2013; 26: 1032-40. 